\title{
Measurement of proton induced Drell-Yan with the polarized nucleon targets at Fermilab-E1039
}

Yoshiyuki Miyachi, Yamagata University

For the E1039 collaboration 


\section{Contents}

- Sivers asymmetry

- Fermilab-E1039

- SeaQuest + Polarized target

- Projection of E1039

- Recent activity

- Time-line

- Summary 


\section{Sivers asymmetry}

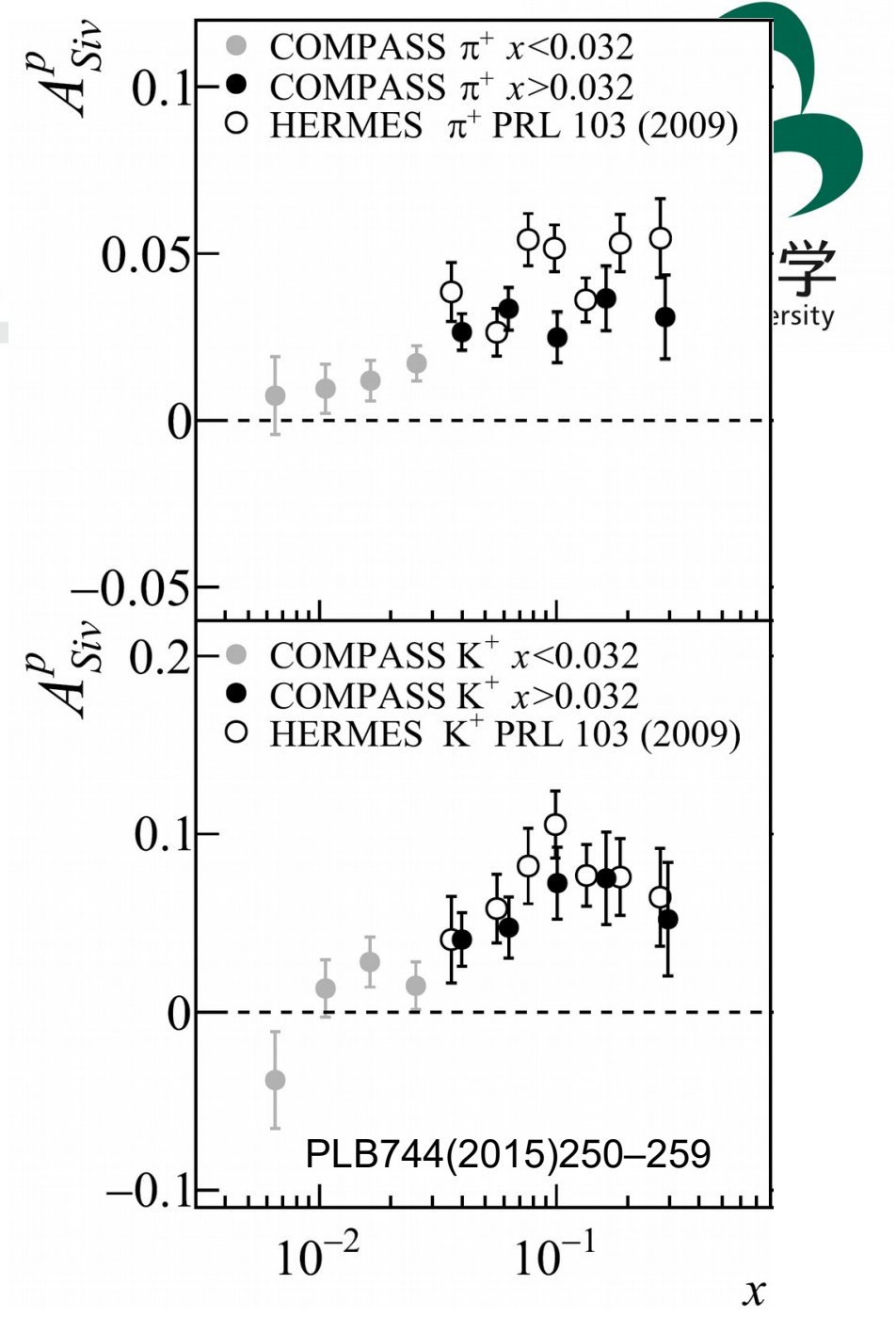

Azimuthal asymmetry of the hadrons from the unpolarized quark inside the transversely polarized nucleon with respected to the nucleon spin

Measured in the DIS experiments HERMES, COMPASS, Jlab $w /$ polarized $p, d$, and ${ }^{3} \mathrm{He}$ 


\section{Sivers TMD distribution}

山形大学

Yamagata University

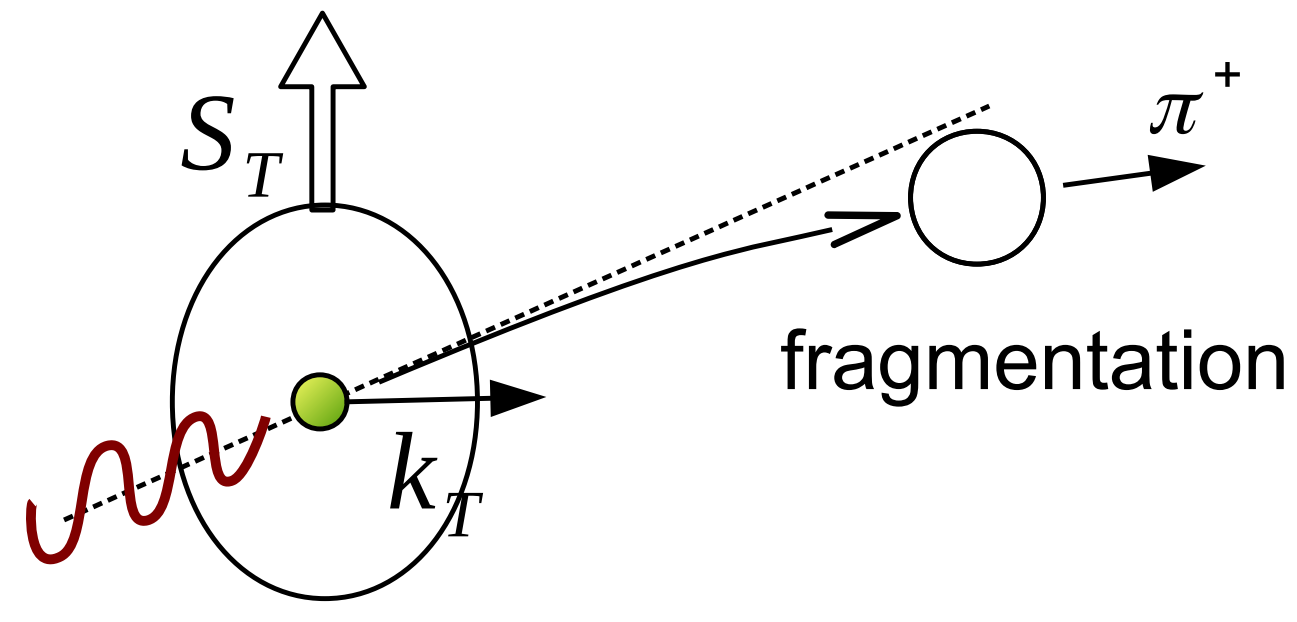

Global analysis of the measured symmetries

$\Rightarrow$ Sivers TMD distribution

M. Anselmino et al., JHEP04 (2017) 046

M. G. Echevarria et al., TMD2P. Sun, F. Yuan, PRD89 (2014) 074013 PRD88 (2013) 114012

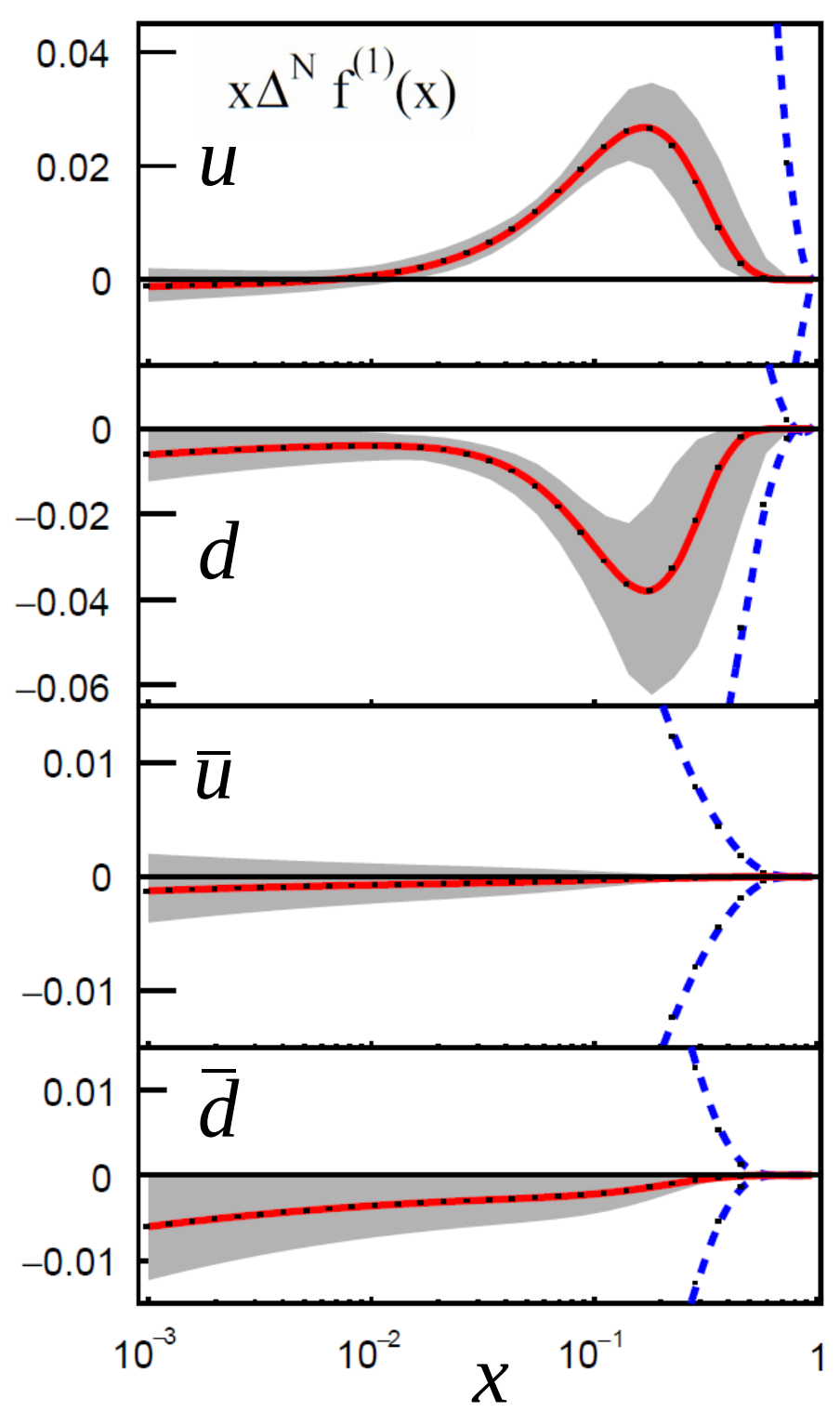




\section{"Non"-universality of Sivers TMD}

DIS $\gamma^{*}+q \rightarrow q^{\prime}$

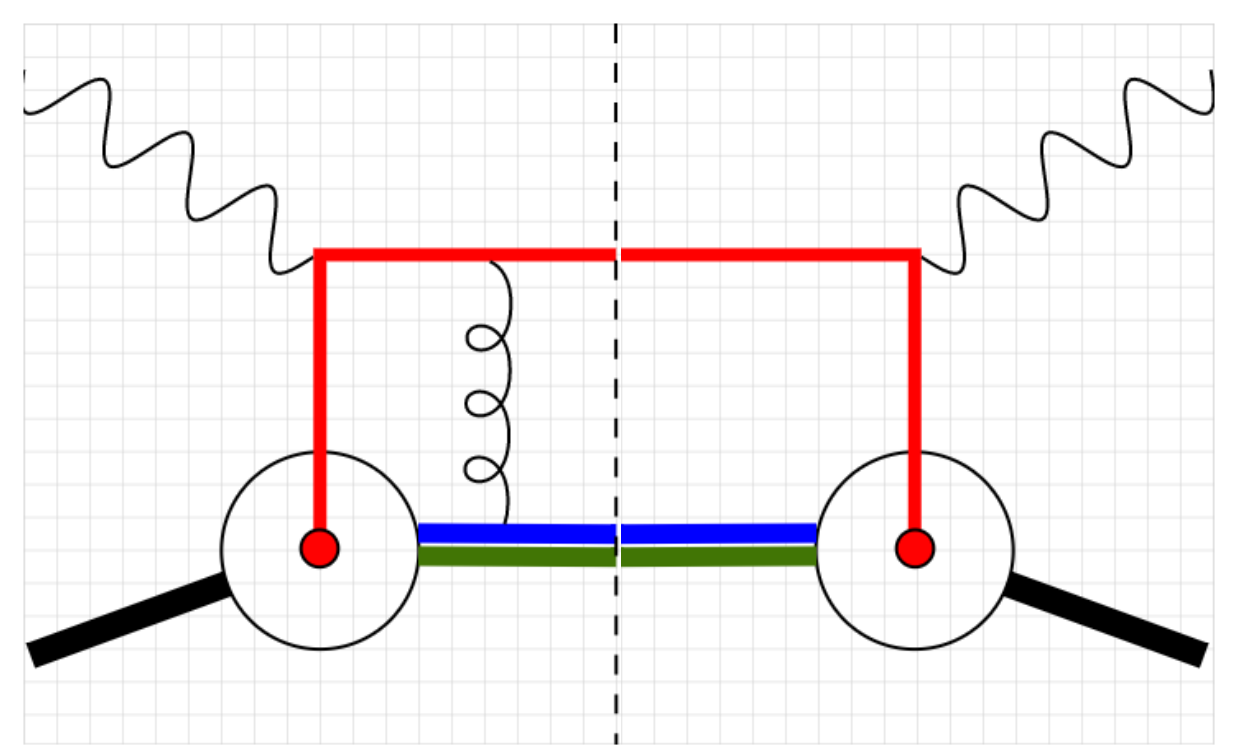

Sivers: Final state interaction

$\rightarrow$ attractive
Drell-Yan $q+\bar{q} \rightarrow \gamma^{*}$

W/Z prod. $q+\bar{q} \rightarrow W / Z$

学

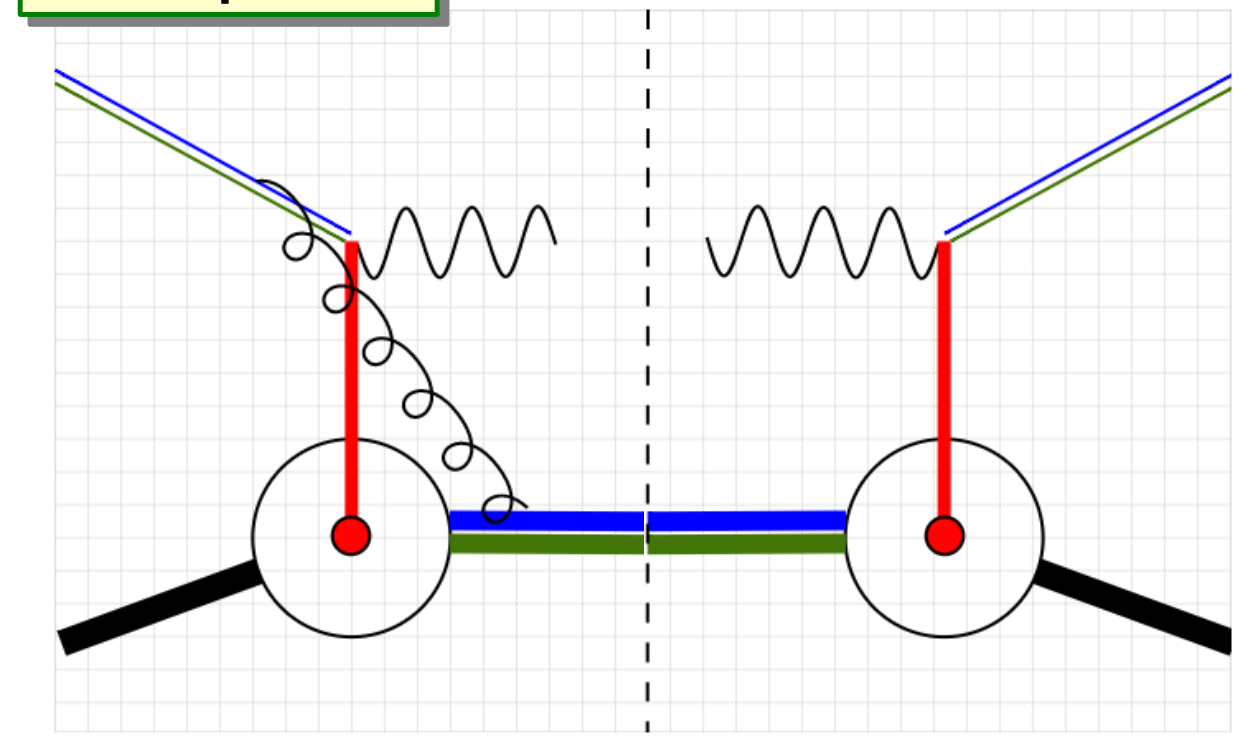

Sivers: Initial state interaction $\rightarrow$ repulsive

$$
\left.f_{1 T}^{\perp}\right|_{\mathrm{DIS}}=-\left.f_{1 T}^{\perp}\right|_{\mathrm{DY}, \mathrm{W} / \mathrm{Z}}
$$


"Non"-universality of Sivers distribution

$$
\left.f_{1 T}^{\perp}\right|_{\mathrm{DIS}}=-\left.f_{1 T}^{\perp}\right|_{\mathrm{DY}, \mathrm{W} / \mathrm{Z}}
$$

- Results from W-prod. @RHIC and Drell-Yan @COMPASS

indicate

the possible sign-change

- It requires

more statistic

to understand $\mathrm{Q}^{2}$ evolution

- TMD framework seems to be OK

The next is "anti-quarks"!
PRL116(2016)132301
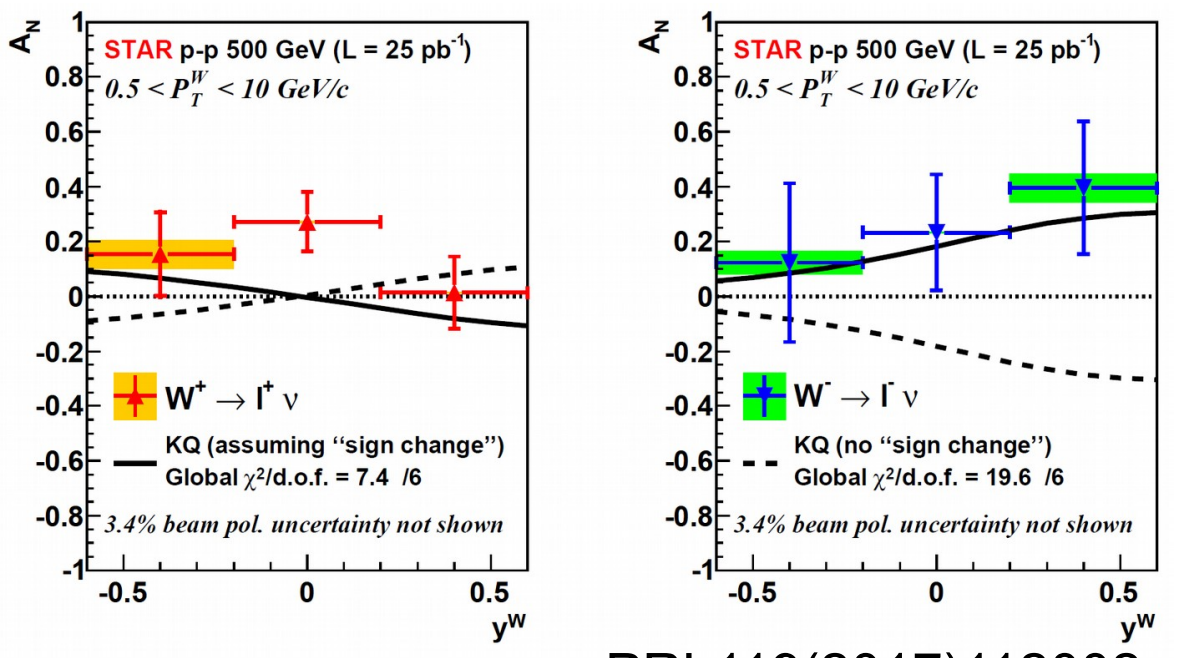

PRL119(2017)112002 


\section{Role of anti-Quarks in the proton spin puzzle}

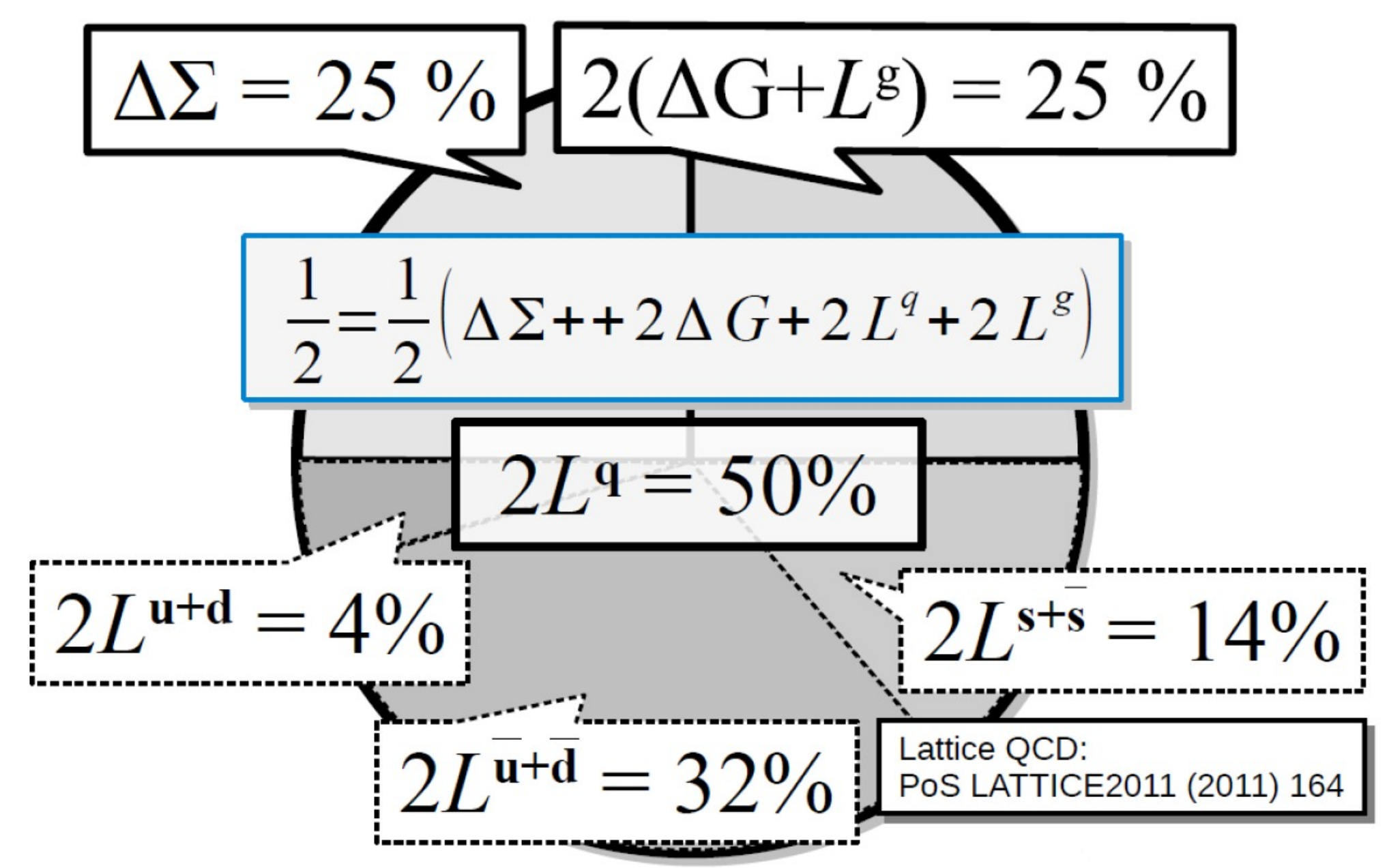




\section{Projection of Sivers asymmetry measurment}

山形大学

Yamagata University

- Proton Beam

- Energy: $120 \mathrm{GeV}(\sqrt{ } \mathrm{s}=15 \mathrm{GeV})$

- Instant luminosity: $4^{*} 10^{35} \mathrm{~cm}^{-2} \mathrm{~s}^{-1}$

- Integrated luminosity: $1.1^{*} 10^{43} \mathrm{~cm}^{-2}$ year $^{-1}$

- Beam time: 2 years

- Mass range $4<\mathrm{M}<8 \mathrm{GeV} / \mathrm{c}^{2}$

- Polarized targets

- Upstream by $2 \mathrm{~m}$ by E906

- Lower $x_{2}$ acceptance

- Better target and dump separation

\section{Drell-Yan Target Single-Spin Asymmetry}

$p p^{\uparrow}\left(d^{\uparrow}\right) \rightarrow \mu^{+} \mu X, 4<M_{\mu \mu}<9 \mathrm{GeV}$

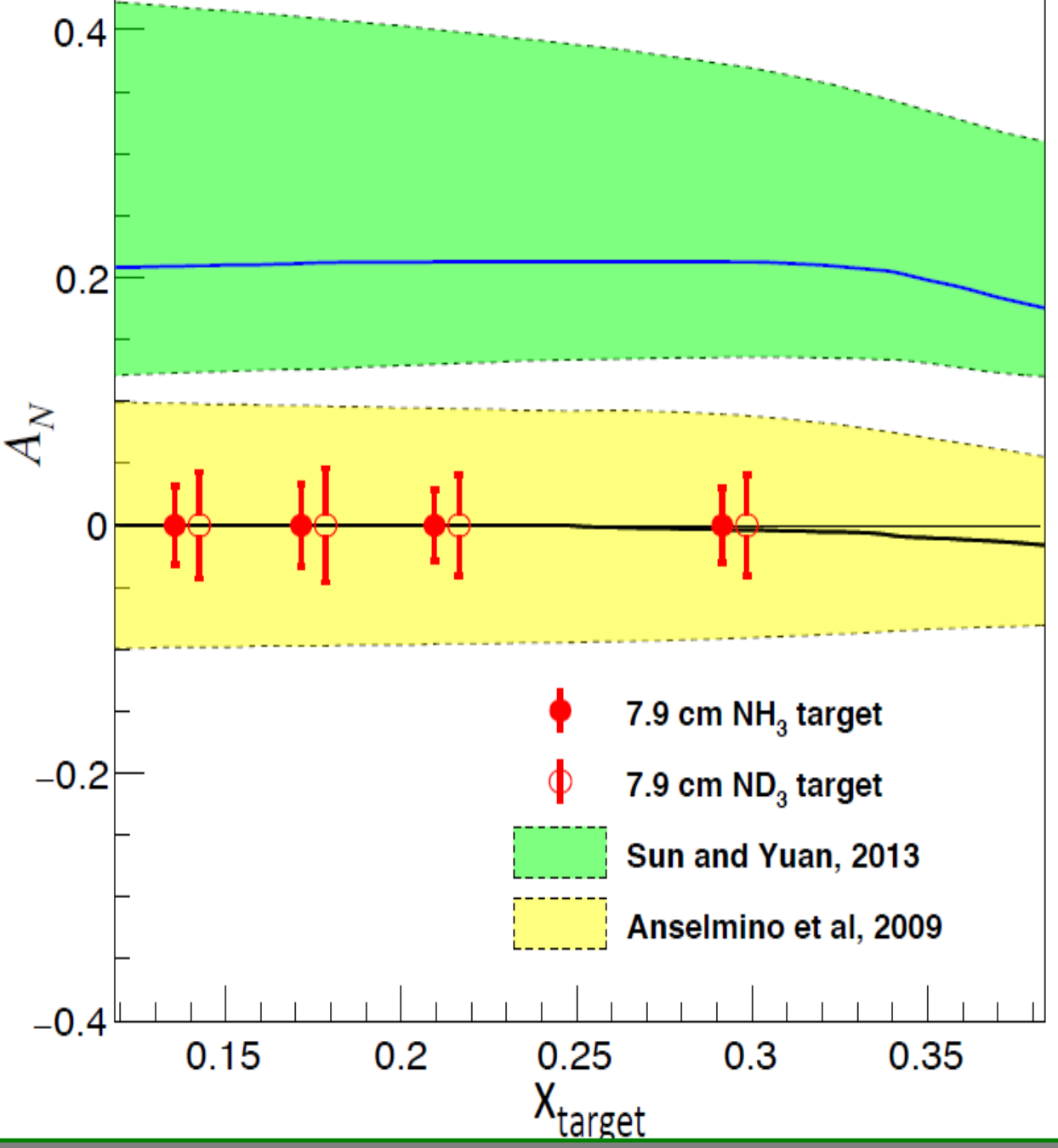

9 


\section{E1039 polarized target}

- $\mathrm{NH}_{3}$ and $\mathrm{ND}_{3}$ targets

- Dynamic Nuclear Polarization

- $1 \mathrm{~K}$ ( ${ }^{4} \mathrm{He}$ refrigerator)

- 5 T magnetic field

- $140 \mathrm{GHz}$ microwave irradiation

- Polarization

90\% for proton

$50 \%$ for deuteron

- Challenge with the high intense proton beam

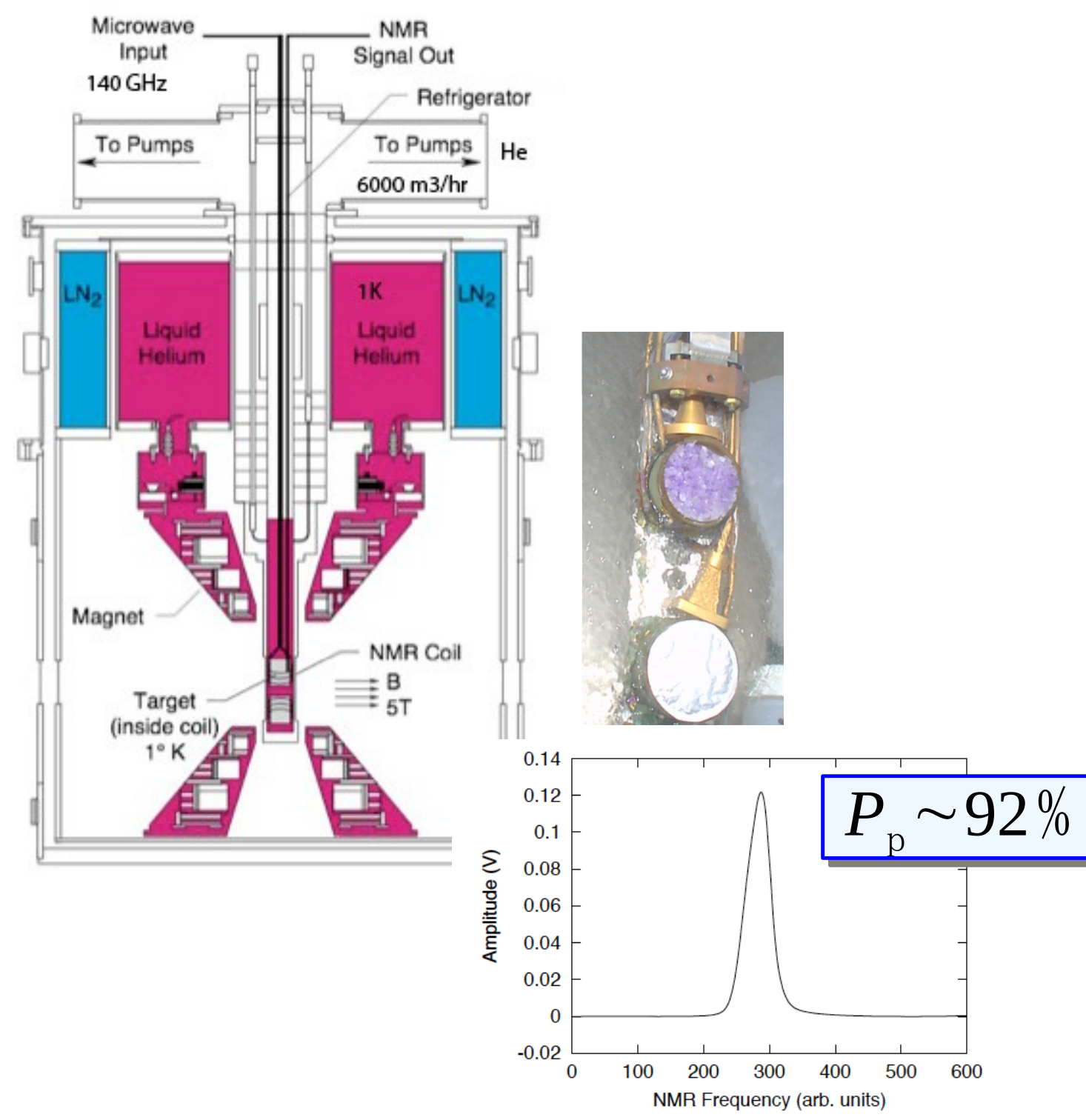




\section{E1039 polarized target}

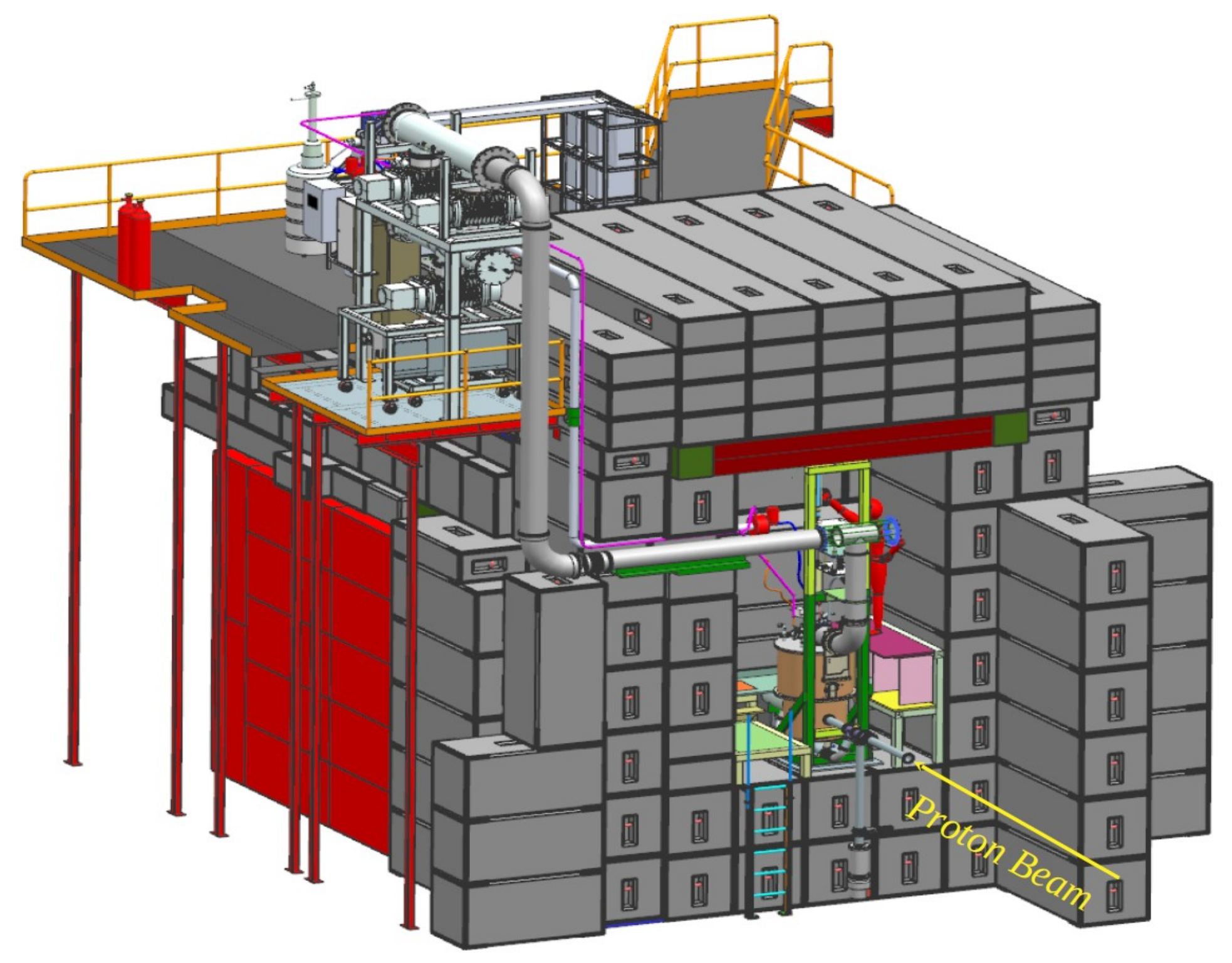




\section{Spectrometer}

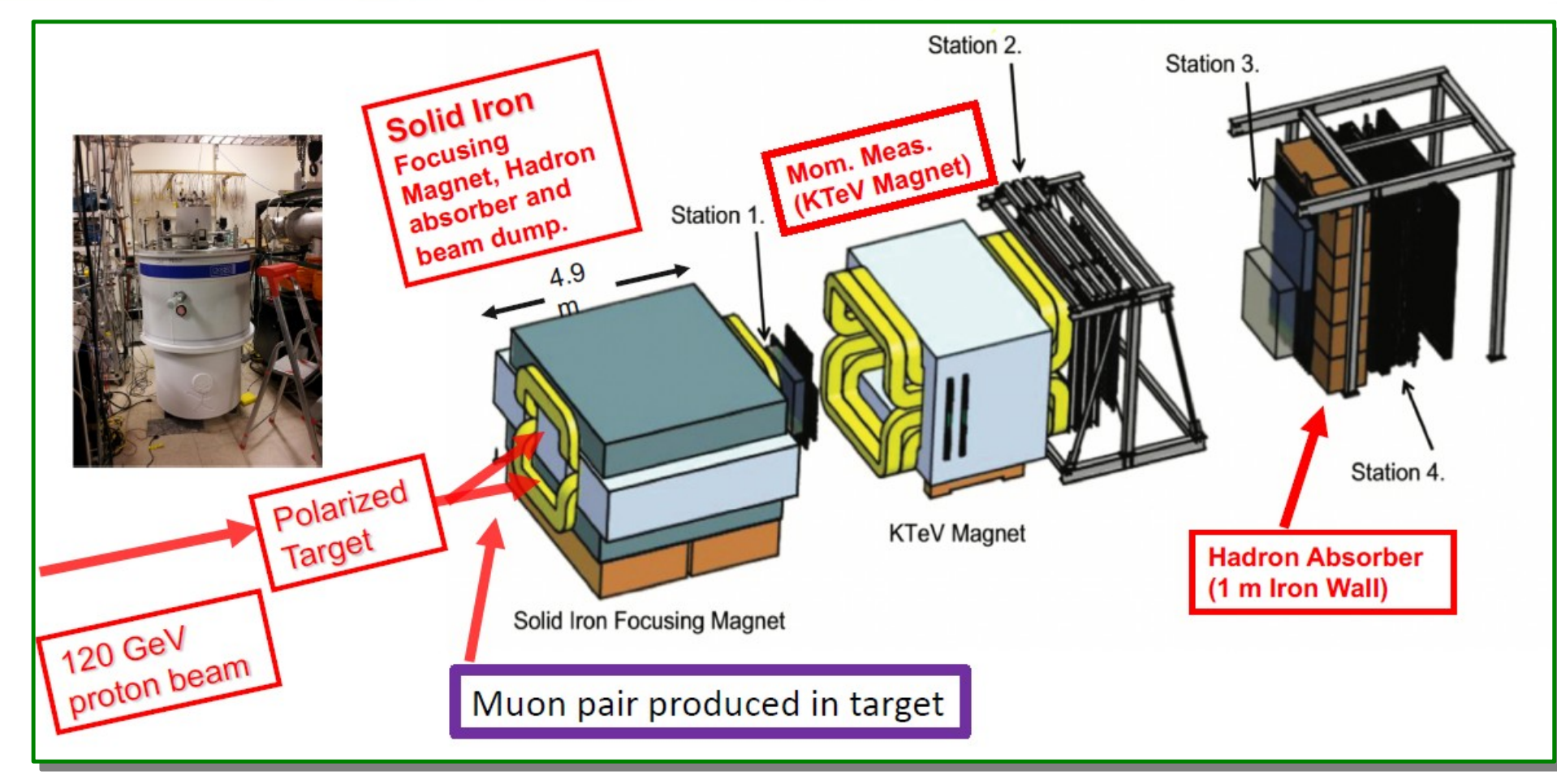

山形大学

- New 90 degree beam monitor in the target area New fiber scintillators are added in the station 1 and 2

- Chamber and hodoscope maintenance and repair fix the known problems from SeaQuest

- DAQ upgrade, Trigger upgrade, ..... 


\section{E1039 Timeline}

\section{- 2018}

- Fermilab $2^{\text {nd }}$-stage approval (2018.05)

- E906 decommissioning

- E1039 commissioning

- New shielding and target platform

- Detector maintenance and repair

- Spectrometer tuning starts at the end of the year

- 2019

- Polarized target installation

- Beam time ( 2 years) 


\section{Summary}

\section{- Fermilab-E1039}

- SeaQuest (anti-quark) + Polarized target (spin)

- Measure Sivers asymmetry in polarized Drell-Yan

- Proving orbiting u-bar, or d-bar ant-quarks inside the polarized nucleon

- Green light on E1039 in 2018

- $2^{\text {nd }}$ stage approval

- from E906 to E1039

- E1039 commissioning is on-going

- 2019

- Beam time!

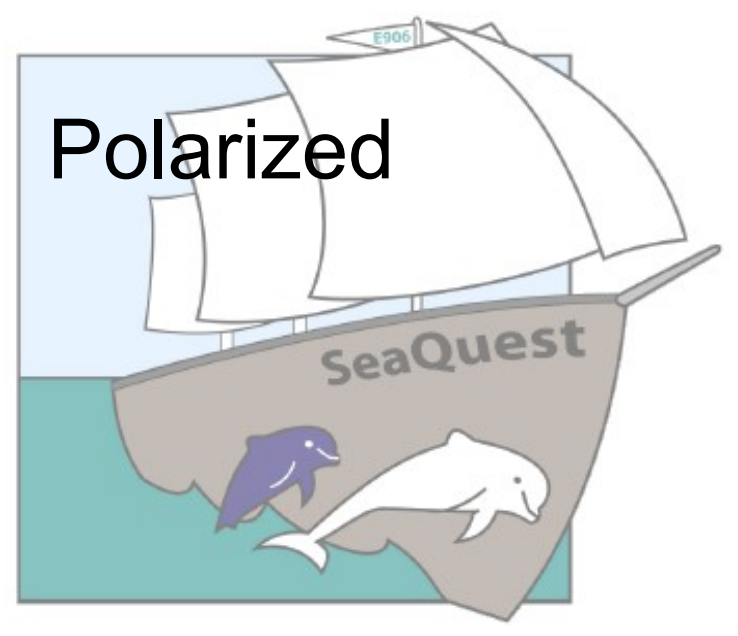

